\title{
Chemical and microstructural analysis of space weathered Fe- sulfides returned from asteroid Itokawa
}

\section{LAURA C CHAVES AND MICHELLE S. THOMPSON}

Purdue University

Presenting Author: 1chavesm@purdue.edu

In their Hayabusa mission, the Japan Aerospace Exploration Agency (JAXA) collected 1534 regolith particles from the stony (S-type) asteroid Itokawa. S-type asteroids have been hypothesized to be the parent bodies of ordinary chondrites. However, S-type asteroids present spectral dissimilarities in the visible near-infrared region (VNIR) compared to ordinary chondrites [1]. These include a lower reflectance (i.e., darkening), an increase of reflectance with increasing wavelength (i.e., reddening), and the attenuation of characteristic bands essentials for mineral identification. These spectral anomalies are thought to be caused by space weathering, which refers to the chemical and microstructural alterations of the minerals on the surfaces of airless bodies as a result of micrometeoroid impacts and solar wind irradiation. Geochemical analysis on olivine, pyroxene, and chromite particles from asteroid Itokawa shows a composition similar to LL4-6 ordinary chondrites [2], verifying the link between stony asteroids and ordinary chondrites.

Space weathering studies have focused on silicate minerals (e.g., olivine, pyroxenes) which are the major constituents of lunar samples and ordinary chondrites. However, sulfide minerals are relevant accessory minerals in ordinary and carbonaceous chondrites, but their contribution to the space weathering of asteroidal surfaces is not well understood yet. To identify the chemical and structural response of sulfide minerals under interplanetary exposure, we performed transmission electron microscopy (TEM), scanning electron microscopy (SEM), and energy-dispersive X-ray spectroscopy (EDS) analyses on two sulfide-bearing Itokawa regolith particles (RBCV-0121 and RC-MD01-0025).

High-resolution transmission electron microscopy (HRTEM) show lattice fringes with d-spacings of $0.56 \mathrm{~nm}$, similar to values for monoclinic pyrrhotite. EDS mapping shows a relative sulfur depletion on the upper $\approx 5 \mathrm{~nm}$ of a sulfide grain from RB-CV0121 content, which has been previously identified on other sulfide Itokawa grains [3] and laser irradiation experiments on troilite [4]. This suggests that space weathering might drive sulfur depletion on the surfaces of airless bodies.

[1] Chapman (2004), Annual Review of Earth and Planetary Sciences 32, 539-67.

[2] Nakamura et al., (2011), Science 333, 1113-1116.

[3] Keller et al., (2014), Earth, Planets and Space 66:71.

[4] Loeffler et al., (2008), Icarus 195, $662-629$. 